\title{
The Application of Color Design in Living Space
}

\author{
Keyu Zhu', a \\ ${ }^{1}$ Taiyuan University of Technology, Taiyuan, Shanxi Province, China \\ a422786254@qq.com
}

Keywords: Color Design, Living Space, Application

\begin{abstract}
Color is a language to express emotion. It is an expression of the human heart in some very complex feelings. Interior color is called "soul", and unlimited color can evoke a sense of the media, it has the inherent power may provoke direct and significant response. It is used in architecture and interior environments are no exception, it can significantly affect the perception of space and form. It is the interior design of the most vivid, the most active factor, plays a decisive role. The color scheme is successful we can meet aesthetic requirements and is personal expression occupants.
\end{abstract}

\section{Introduction}

From ancient Greece to the present, the history of the color continuum concept showing people of color continued fascination with architecture and design as well as the environment of a natural color update process. In the 21st century, color as an important factor in the overall design of the part, and other design form language as there are aesthetic and practical dual role. Colors that can decorate and beautify things arouse people's interest and love, but also to adjust the visual effect of things functional, allowing users to feel the physical and psychological sense of unity and better balance [1].

Unlimited colors can evoke a sense of the media, it has inherent power can provoke immediate and significant response, and thus it has become a symbol of the natural and man-made world languages [1]. It is used in architecture and interior environments is no exception, it can significantly impact on the architecture and interior space and form perception.

Today in architecture, interior and urban space planning and design process color has become a very important part of the color with the gradual emphasis on educational institutions and professional organizations became a professional design research, education and application in color have a more for the broad development. In this case, the color is also more important: whatever the tune (with good color tuning) we feel pleasure, who would not join the tone of color we feel glare or bored [2]. This color will feel aesthetic judgments associate art color performance we can find the importance of color in our lives. Spread the development of modern business culture and industrial culture, so that the color and design thinking as one, greatly contributed to the economic prosperity and cultural development. In this development, based on the use of color closer to the deep nature of the design, thereby improving the quality of people's lives.

\section{The Relationship of Color and Interior Design}

Interior color is an integral part of interior design. In the interior color design, the use of effective means to color theory and interior design convergence, to create an atmosphere of indoor and convey the psychological feeling to the user plays a crucial role (showed in Figure 1) [2]. Taking advantage of people of color overall visual experience, to create rich layers, personality, order and good atmosphere environment, so as to achieve a multiplier effect. Slaughter within the design of the most vivid, the most active factor is the color of luck claw, or knowledge to create heat, or calm, or elegant atmosphere. 


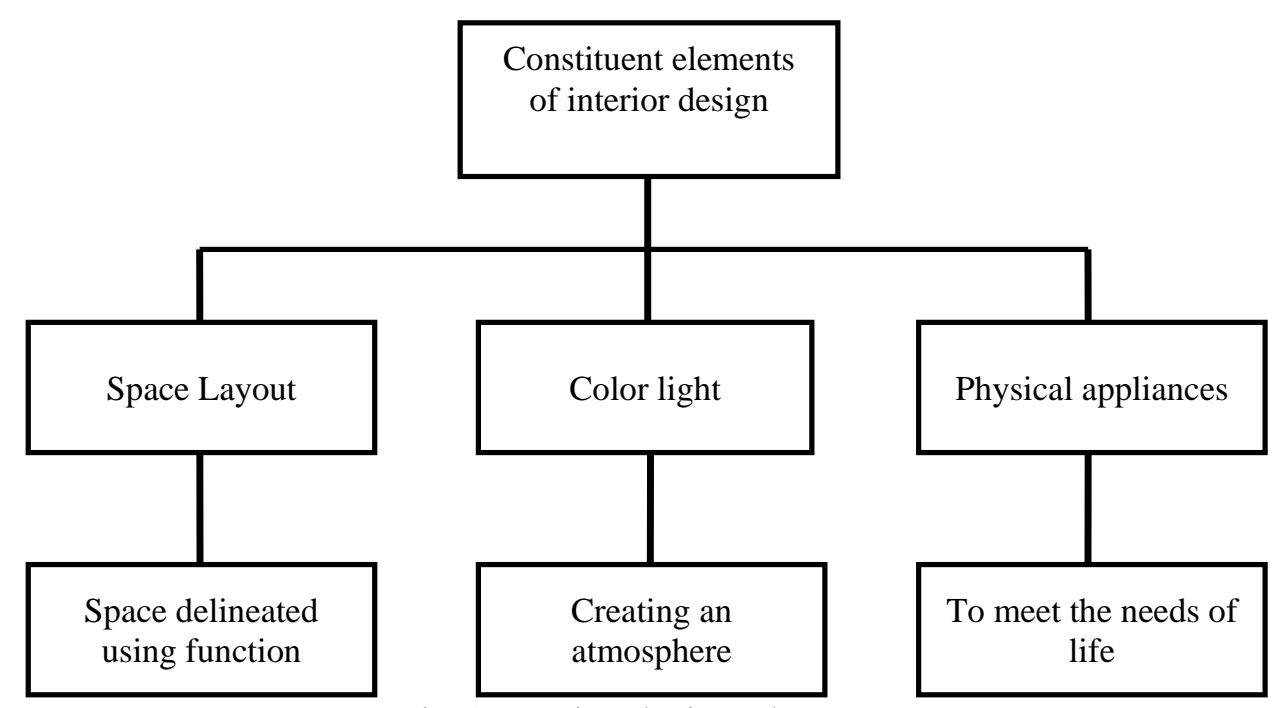

Fig.1 Interior design elements

On home design, in determining the overall color design style after they entered the stage. Interior color constitutes include keynote color, secondary color and decorative colors [1,3]. First, the tone of color (or background color) refers to ceiling, wall and floor colors, seventy percent of their area's largest, accounting for the overall color, but also accounted for the largest part of the interior space, generally set off from furniture the role of equipment and furnishings pieces, etc., it should consider choosing long-term use will not make eye fatigue possessed overall sense of color. Among them, the largest area of the wall color, the overall atmosphere of the room to play a decisive role. Smarter pale hue can expand visual sense of space, low brightness color will make the space feel cramped. Warm with a sense of expansion, but the warm light reflectance properties could easily lead to visual fatigue, so use caution [4]. Home ground is usually decorated with floor tiles or carpet; their color will affect the overall interior color impression. Ground colors can be chosen to coordinate with the curtains, sofas and other fabric colors, too bright or dim may cause visual stimulation or mental fatigue human senses, should avoid a large area [2]. Ceiling color should be used in conjunction with the wall and floor, warm colors can make sense to reduce the visual ceiling, and cool smarter hue can be improved sense of space. In the interior, the design in the form of the top surface is often the most important design elements, it may be a natural reaction to the building structure system, it can also be separated from the structure to become a positive factor in a visual space. Secondly, the secondary color (or body color) including furniture, curtains, etc., about twenty-five percent of the overall space, played by wall color background, but also said that the role entrusted to build other indoor devices, with the maintenance of tone color and embellishment color balance, control room atmosphere functions. Furniture occupies a large area in the interior space, furniture, color is determined, taking into account the color to walls, floors, doors, windows and other furnishings coordinated with it, can be given the space to emotion [5]. Third, decorative colors, color scheme refers to decorative pieces of furnishings. They are indoor landscaping skit, play a coordinating role in the overall color space. Flexible furnishings and colorful greenery form morphology of India lining constitute the interior color highlights.

\section{Interior Design with Color Configuration}

Emphasize the color of the master-slave relationship. In the color scheme, only the same simple and distinct relationship, it can not constitute a harmony of color, the color should have a clear master-slave relationship [3]. Main color is determined according to the product's functionality, color symbolic. Other color was subordinate color, at the non-mainstream status in the area and the location cannot be overwhelming, affecting melody.

Maintaining mental balance color vision. Visual psychological balance mainly refers to form neural dynamics of human balance and balance of color vision. Light stimuli directly affects the physical immediacy of the psychological effects of color from the color of human physiology 
occurred [4]. Color should be visual mental balance principle to grasp hue, lightness and purity. People's eyes will appear red too much green afterimage, if you will see bright colors too dark afterimage appears, this is the result because of the visual physiological imbalance. The eyes see various color neutral gray or cooperate with each other properly, it will not appear visual afterimage, indicating that visual state at the moment is balanced. Experience has shown that: middle gray produce a fully balanced state in visual psychology, which is also the product design using the gray ones for the sake of color.

Highlight color of the symbolic significance. Further sublimation color emotions, that it can profoundly express people's ideas of faith, which is the color symbolic significance. In every corner of the world today, at every level of society, there will be a wide range of products. Different nationalities, different regions, different cultural backgrounds, different levels of consumption, demand for products will have different differences [5]. Color some products here because wealthy certain symbolic significance, popularity, but in another place on the contrary, the symbolic color language, both personality have in common. Designers should not personal preferences to determine the color, it should address the specific situation in-depth understanding of their different symbolic meaning, the appropriate use of symbolic elaboration ideal color scheme.

Pay attention to the color of the order of. Order is the soul of all the plastic arts. Hard to imagine a chaotic product will become a successful classic [4]. The color of the order of performance on the one hand, the color relationship, that certain geometric relationship in color hue ring, on the other hand also in the logic of the project itself color plans. Today, many enterprises implement serialization, family-oriented design, the color of their projects also particularly important product order. Clever use of color can order products showed rhythmic beauty, rhythm US order US order and balance.

Beauty care color coordination. The United States is seeking to coordinate a unified color by changing the color scheme is obtained, namely the color hue, lightness and purity, both to find its similarities [6], but also reflect the partial color by comparing characteristics; both the parts its own rhythm, but also make the various parts of harmony and unity, so that the color space of the overall coordination of the rich beauty.

\section{Color Design in Living Space}

Faced with changing color space and intricate relationship, to get the overall coordination, must follow the universal law and the general rules of color in interior design [6]. A mix of color phenomenon, from the configuration rules, can be summarized as the same color configuration, the difference in the color configuration, contrasting color configuration, there is no color line color line and configuration, and they each have their own characteristics in the color design of a room [4].

Used in interior design contrast color configuration: you can highlight the indoor overall tone, rendering strong, bright, lively, joyful emotion atmosphere; you can use striking, eye-catching contrast color, focus space standing room [4], the focus area; you can use a small area of contrasting colors accessories, indoor play a painted dragon points clear of decoration; may also adjust the color brightness moderately poor, the purity of the poor contrast the strength to control the size, reach performance indoor purposes.

Indoor house, function and purpose of each space is not the same, we must to consider interior design and use of color depending on the properties. Not only have that, but also pay attention to the same association between the characteristics of the room, adjusted the sense of coordination between regions [5]. It can also be a further building exterior and interior design linked to achieve the overall unity, the parts related and have their own characteristics. Performing residential interior color design, according to interior features and functions will be divided into three zones [6].

\section{Public areas (entrance hall / hallway / living / dining, etc.)}

Public areas are all family members will use the space, but also the outside world, the guests will visit the region. Making the interior color to consider when designing the selected tone with a certain public, not only accepted by the public, but also reflects the family's unique image and style, such as Fig2. 

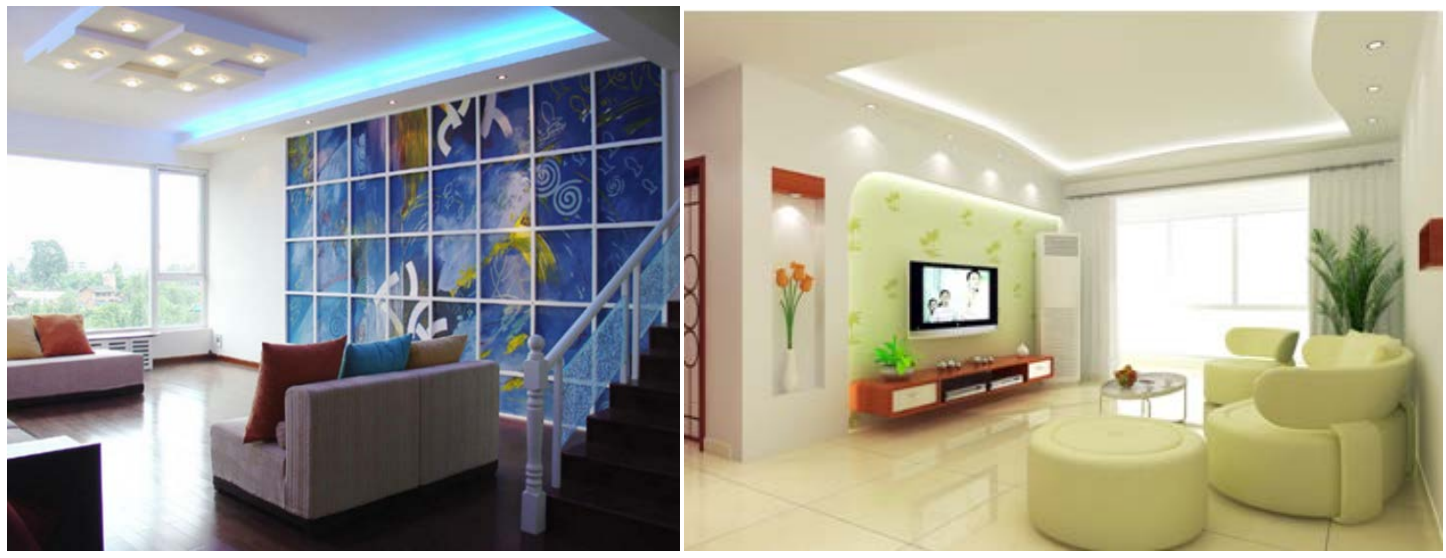

Fig.2 color design of public areas

\section{Private areas (primary and secondary bedroom / children's room / cloakroom / den / activity room)}

Dedicated areas can be used in preference to decide what color to match the color design space according to each user's preferences. Features and nature of the region itself to make the color space designed with individual characteristics, such as Fig 3.

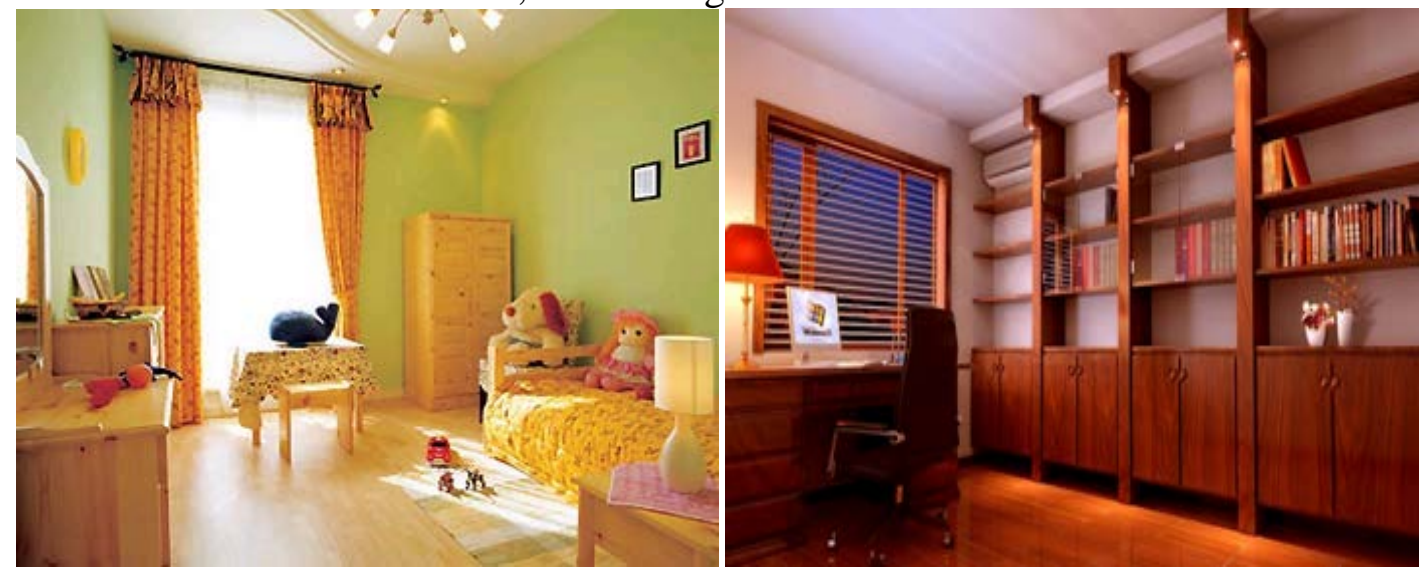

Fig.3 color design of private areas

\section{Assisted areas (kitchen / bathroom / toilet / bathroom / storage room, etc.)}

Secondary zones are mainly space with water or fire. Water, fire, health, ease of maintenance and other functions are necessary conditions. When designing in this area and bright, pure is the best color matching results, such as Fig 4.

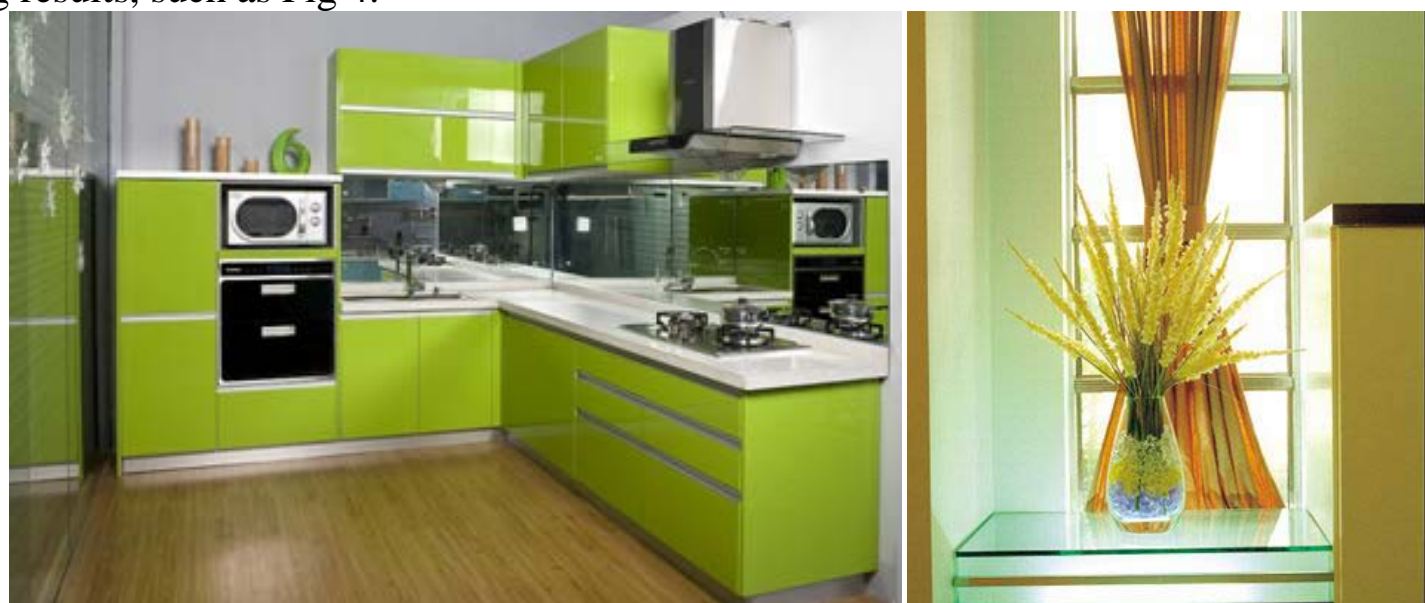

Fig.4 color design of assisted areas

\section{Conclusion}

Color design interior design is a very important part of the focus of the design is how to obtain vivid colors, in order to meet the dual physical and psychological needs of the people. In view of the 
importance and difficulty factor color color research, the study of indoor environment color design approach is always the focus of attention of design theory. However, since a long time, due to the personnel engaged in the study of indoor color is always dependent on the reference plane sided color research, which was directly applied in an indoor environment, but did not notice the presence in the indoor environment is "Space + Time" the four-dimensional space-time relationship with the two-dimensional plane are essentially different, so the color of the indoor environment research has not been significant progress.

\section{References}

[1] J. Chen, Double hair design color, Southeast University Press, 2012.pp.52-54.

[2] M.G. Du and X.P. Ling, On the color scheme used in the indoor environment. Jiaxing University, 2005, pp83-87.

[3] K.Y. Yu and D.Sh Quan, Interior design study based on technical performance. Tongji University master's degree thesis, 2005,pp.38-42.

[4] S.S.Wen, Built Environment Color Design, Beijing: China Building Industry Press, 2009, pp. 45-47.

[5] J. Chen, Construction of architectural form and explore ways of comparison, China Building Industry Press, 2013.pp.22-26.

[6] Y.J. Li, Sh.T. Shi, Color design of Living Environment, China Building Industry Press, 2012.pp.11-15. 